\title{
Alain Billionnet \\ Brève communication. Transformation du problème de partitionnement en un problème d'ensemble stable de poids maximal
}

Revue française d'automatique, d'informatique et de recherche opérationnelle. Recherche opérationnelle, tome 12, nº 3 (1978), p. 319-323.

<http://www.numdam.org/item?id=RO_1978_12_3_319_0>

(C) AFCET, 1978, tous droits réservés.

L'accès aux archives de la revue « Revue française d'automatique, d'informatique et de recherche opérationnelle. Recherche opérationnelle » implique l'accord avec les conditions générales d'utilisation (http://www.numdam.org/ legal.php). Toute utilisation commerciale ou impression systématique est constitutive d'une infraction pénale. Toute copie ou impression de ce fichier doit contenir la présente mention de copyright.

\section{Numdam}




\title{
Brève communication
}

\section{TRANSFORMATION \\ DU PROBLĖME DE PARTITIONNEMENT \\ EN UN PROBLĖME D'ENSEMBLE STABLE \\ DE POIDS MAXIMAL ( ${ }^{*}$ )}

\author{
par Alain BILLIONNET $\left({ }^{1}\right)$
}

\begin{abstract}
Résumé. - Nous montrons, dans cet article, que le problème de partitionnement peut se poser comme la détermination de l'ensemble stable, de poids maximal, dans un graphe. Cette approche permet d'envisager la résolution du problème de partitionnement par un algorithme de graphes et conduit à une formulation inhabituelle de ce problème.
\end{abstract}

\section{INTRODUCTION}

Considérons le problème de partitionnement $\mathrm{PP}$ :

$$
\text { minimiser } z=c x \text {, }
$$

avec

$$
A x=e, \quad x_{j}=0 \text { ou } 1,
$$

où $A=\left(a_{i j}\right)$ est une $m \times n$ matrice donnée et telle que $a_{i j}=0$ ou $1 ; e$, un $m \times 1$ vecteur dont tous les éléments sont égaux à $1 ; c$, un $1 \times n$ vecteur à coefficients positifs connus; $x$, le vecteur $n \times 1$ des inconnues $x_{j}$.

Nous montrons, ici, que la résolution du problème PP peut se ramener à la recherche de l'ensemble stable de poids maximal (e.s.p.m.) dans un graphe et nous en déduisons un problème équivalent $\mathrm{PP}^{\prime}$.

\section{GRAPHE ASSOCIÉ AU PROBLÈME PP}

Associons aux données du problème PP le graphe simple $G=(X, V)$ défini de la façon suivante :

$$
\begin{gathered}
X=\left\{x_{1}, x_{2}, \ldots, x_{n}\right\} \\
{\left[x_{r}, x_{s}\right] \in V \Leftrightarrow \quad \exists i \in\{1,2, \ldots, m\} \text { tel que } a_{i r} . a_{i s}=1 .}
\end{gathered}
$$

Appelons $p_{1}\left(x_{j}\right)=\sum_{i=1}^{i=m} a_{i j}$ le poids du sommet $x_{j}$.

(*) Reçu septembre 1977.

(1) Institut d'Informatique d'Entreprise-C.N.A.M.

R.A.I.R.O. Recherche opérationnelle/Operations Research, vol. 12, nº 3, août 1978 


\section{PARTITIONNEMENT ET STABILITÉ}

Considérons d'abord le problème réduit qui consiste à trouver un partitionnement sans tenir compte de la fonction économique.

LeMme 1 : Le problème $A x=e$ admet une solution si et seulement si il existe, dans $G$, un ensemble stable, $S$, de poids $m$. Cette solution est alors définie de la façon suivante : $x_{j}=1 \Leftrightarrow x_{j} \in S$.

Preuve : a) condition nécessaire. Considérons une solution $s$ du problème $A x=e: x_{j_{i}}=x_{j_{2}}=\ldots=x_{j p}=1, \quad x_{j_{p+1}}=x_{j_{p+2}}=\ldots=\dot{x}_{j_{n}}=0$. Soit $S$ le sousensemble de $X$ formé des sommets $x_{j_{1}}, x_{j_{2}}, \ldots, x_{j_{p}} ; \forall k, l \in\{1,2, \ldots, p\}$, $\left[x_{j_{k}}, x_{j_{l}}\right] \notin V . S$ est donc stable. Soit $E_{j}, j=1,2, \ldots, n$, l'ensemble des équations où apparaît la variable $x_{j}$ :

$$
P_{1}(S)=p_{1}\left(x_{j_{1}}\right)+p_{1}\left(x_{j_{2}}\right)+\ldots+p_{1}\left(x_{j_{p}}\right)=\left|E_{j_{1}}\right|+\left|E_{j_{2}}\right|+\ldots+\left|E_{j_{p}}\right| .
$$

On a donc $P_{1}(S)=m$ puisque $E_{j_{2}}, E_{j_{2}}, \ldots, E_{j_{1}}$ forment une partition de $E$ (ensemble des équations du problème);

b) condition suffisante. Soit $S=\left\{x_{j_{1}}, x_{j_{2}}, \ldots, x_{j_{p}}\right\}$ un ensemble stable de poids $m$. Les ensembles $E_{j_{1}}, E_{j_{2}}, \ldots, E_{j_{p}}$ sont deux à deux disjoints et forment une partition de $E$ puisque $\left|E_{j_{1}}\right|+\left|E_{j_{2}}\right|+\ldots+\left|E_{j_{p}}\right|=m$. On en déduit que $x_{j_{1}}=x_{j_{2}}=\ldots=x_{j_{p}}=1, x_{j_{p+1}}=x_{j_{p+2}}=\ldots=x_{j_{n}}=0$ est bien une solution de $A x=e$.

Lemme 2: Le poids de tout ensemble stable de $G$ est inférieur ou égal à $m$.

Preuve: Supposons qu'il existe un ensemble stable

$$
S_{0}=\left\{x_{j_{1}}, x_{j_{2}}, \ldots, x_{j_{q}}\right\} \text { tel que } P_{1}\left(S_{0}\right)>m .
$$

$P_{1}\left(S_{0}\right)>m \Rightarrow \exists k$ et $l(k \neq l) \in\{1,2, \ldots, q\}$ tels que $E_{j_{k}} \cap E_{j_{l}} \neq \emptyset$;

$E_{j_{k}} \cap E_{j_{l}} \neq \emptyset \Rightarrow\left\{x_{j_{k}}, x_{j_{l}}\right\} \in V$ ce qui est contraire à l'hypothèse.

On déduit immédiatement des lemmes 1 et 2 les deux théorèmes :

THÉORÈME 1 : Si le problème $A x=e$ admet une solution, à tout e.s.p.m. du graphe $G$ correspond une solution de $A x \doteq e$ et réciproquement.

ThÉORÈme 2 : Si le problème $A x=e$ n'admet pas de solution, le poids de tout ensemble stable de $G$ est inférieur à $m$.

\section{MODIFICATION DU SYYSTÈME DE POIDS ASSOCIÉ A $G$}

En fait, on ne cherche pas une solution quelconque de $A x=e$ mais celle qui minimise $z$. Il faut donc sélectionner parmi tous les e.s.p.m. de $G$ celui qui 
correspond au minimum de $z$. Modifions, pour cela, le système de poids associé à $G$. Appelons $p_{2}\left(x_{i}\right)$ le nouveau poids du sommet $x_{j}$ :

$$
p_{2}\left(x_{j}\right)=L \cdot p_{1}\left(x_{j}\right)-c_{j},
$$

$L$ étant un entier supérieur à $\sum_{j=1}^{n} c_{j}$. Appelons respectivement $\mathscr{S}_{1}$ et $\mathscr{S}_{2}$ les deux systèmes de poids fondés sur le calcul de $p_{1}\left(x_{j}\right)$ ou $p_{2}\left(x_{j}\right)$. Nous pouvons alors démontrer que :

LEMME 3 : Tout e.s.p.m. dans le système $\mathscr{P}_{2}$ est un e.s.p.m. aans le système $\mathscr{S}_{1}$ (la réciproque n'est pas vraie).

Preuve : Soit $S$ un e.s.p.m. dans $\mathscr{S}_{2}$ :

$$
P_{2}(S)=\sum_{x_{j} \in S}\left[L \cdot p_{1}\left(x_{j}\right)-c_{j}\right]=L \cdot P_{1}(S)-\sum_{x_{j} \in S} c_{j} .
$$

Supposons que $S$ ne soit pas de poids maximal dans $\mathscr{S}_{1}$, c'est-à-dire qu'il existe un ensemble stable $S_{0}$ tel que $P_{1}\left(S_{0}\right)>P_{1}(S)$ :

donc

$$
P_{2}\left(S_{0}\right)-P_{2}(S)=L \cdot\left[P_{1}\left(S_{0}\right)-P_{1}(S)\right]-\sum_{x_{j} \in S_{0}} c_{j}+\sum_{x_{j} \in S} c_{j} ;
$$

$$
P_{2}\left(S_{0}\right)-P_{2}(S) \geqq L-\sum_{x_{j} \in S_{0}} c_{j}+\sum_{x_{j} \in S} c_{j},
$$

et, puisque $L>\sum_{j=1}^{n} c_{j}, P_{2}\left(S_{0}\right)-P_{2}(S)$ est positif, ce qui est contraire à l'hypothèse.

Nous déduisons aisément du lemme 3 et du théorème 1 le théorème suivant :

THHÉORÈME 3 : Si le problème $A x=e$ admet une solution, à l'e.s.p.m., dans le système $\mathscr{S}_{2}$, correspond une solution de $A x=e$.

D'après cé dernier théorème, nous pouvons faire correspondre à l'e.s.p.m., dans le système $\mathscr{S}_{2}$, une solution, $s$, de $A x=e$. Montrons que cette solution est celle du problème $\mathrm{PP}$, ce qui permet d'énoncer :

THÉORÈme 4 : Si le problème PP admet une solution, l'e.s. p.m. du graphe G, dans le système $\mathscr{S}_{2}$, correspond à cette solution.

Preuve: Soit $S$ un e.s.p.m. dans le système $\mathscr{S}_{2}$.

Supposons qu'il existe une solution $s_{0}$ de $A x=e$, avec $z\left(s_{0}\right)<z(s)$. Soit $S_{0}$ l'ensemble stable correspondant à cette solution :

$$
P_{2}\left(S_{0}\right)=\sum_{x_{j} \in S_{0}}\left[L \cdot p_{1}\left(x_{j}\right)-c_{j}\right],
$$

vol. $12, \mathrm{n}^{\circ} 3$, août 1978 


$$
P_{2}\left(S_{0}\right)-P_{2}(S)=L . m-\sum_{x_{j} \in S_{0}} c_{j}-L . m+\sum_{x_{j} \in S} c_{j}>0,
$$

ce qui est contraire à l'hypothèse.

Le théorème 4 permet d'envisager la résolution du problème de partitionnement par un algorithme de graphes. Cet algorithme devra tenir compte, pour être performant, d'une part des caractéristiques générales de l'e.s.p.m. d'un graphe et, d'autre part, des propriétés particulières que possède l'e.s: p.m. associé au problème de partitionnement.

\section{AUTRE FORMULATION DU PROBLẼME PP}

On déduit facilement du théorème 4 que, dans le cas où $\mathrm{PP}$ admet une solution, les deux problèmes $\mathrm{PP}$ et $\mathrm{PP}^{\prime}$ sont équivalents (ont le même ensemble de solutions) :

$$
\begin{aligned}
& \text { PP } \\
& \text { minimiser } z=\sum_{j=1}^{n} c_{j} x_{j} \\
& \text { avec } \sum_{j=1}^{n} a_{i j} x_{j}=1(i=1,2, \ldots, m) \text { avec } \sum_{j=1}^{n} a_{i j} x_{j} \leqq 1 \quad(i=1,2, \ldots, m) \text {, } \\
& x_{j}=0 \text { ou } 1 . \\
& x_{j}=0 \quad \text { ou } \quad 1 \\
& \text { où } \quad c_{j}^{\prime}=L \cdot \sum_{k=1}^{m} a_{k j}-c_{j} \\
& \text { et } \quad L>\sum_{j=1}^{n} c_{j} \text {. } \\
& \text { maximiser } z=\sum_{j=1}^{n} c_{j}^{\prime} x_{j}
\end{aligned}
$$

\section{BIBLIOGRAPHIE}

1. C. BeRge, Graphes et hypergraphes, Dunod, Paris, 1970.

2. J. Delorme et E. Heurgon, Problèmes de partitionnement : exploration arborescente ou méthode de troncatures? R.A.I.R.O., V-2, juin 1975, p. 53-65.

3. G. Demoucron, Ensembles stables intérieurement d'un graphe, Gestion, juillet/août 1968.

4. R. Faure et Y. Malgrange, Une méthode booléienne pour la résolution des programmes linéaires en nombres entiers, Gestion, avril 1963.

R.A.I.R.O. Recherche opérationnelle/Operations Research 
5. R. S. Garfinkel et G. L. Nemhauser, The Set Partitionning Problem: Set Covering with Equality Constraints. Opns. Res., vol. 17, 1969, p. 848 à 856.

6. R. S. Garfinkel et G. L. Nemhauser, Integer Programming, chap. 8, John Wiley and Sons, 1972.

7. M. Gondran, Les problèmes de partition et de recouvrement: applications et algorithmes, E.D.F. Bulletin de la Direction des Études et Recherches, série C, Mathématiques-Informatique, $\mathrm{n}^{\circ} 2$, 1976, p. 59-68.

8. M. Gondran et J. L. Lauriere, Un algorithme pour le problème de partitionnement, R.A.I.R.O., V-1, 1974, p. 25-38.

9. B. Roy, Algèbre moderne et théorie des graphes, t. 2, chap. 6, Dunod, Paris, 1970. 